\title{
Reconfigurable Fault Tolerant Flight Control based on Nonlinear Model Predictive Control
}

\author{
D. K. Kufoalor and T. A. Johansen
}

\begin{abstract}
Constrained Nonlinear Model Predictive Control (NMPC) is shown to have potentials for reconfigurable fault tolerant control of highly nonlinear, intrinsically unstable, high performance aircraft. Results on fault tolerance of NMPC autopilots were obtained for an F-16 fighter aircraft model, without the implementation of any prestabilizing controllers. It has been shown that NMPC has inherent fault detection capabilities due to its effective utilization of feedback and its internal model predictions. Actuator (control surface) faults, including extreme cases of total actuator failure are examined as test cases for the NMPC reconfigurable fault tolerant control scheme developed in this work. The NMPC autopilots implementation and simulations were done using the ACADO nonlinear optimization solver.
\end{abstract}

\section{INTRODUCTION}

The increasing complexity of modern and future flight control systems demands advanced and reliable control techniques that will ensure safety and survivability. When faults occur, it is very important that system stability is maintained and an acceptable system performance is attained.

The reconfigurable fault-tolerant control systems review in [1] highlights important limitations on conventional approaches to solving reconfigurable control problems for constrained multivariable systems and systems with significant nonlinearities. In general, how to design fault-tolerant control systems which can work effectively in the entire range of nonlinear systems, and how to distinguish the changes induced by faults from that by operating condition variations is still a challenge.

In the past few decades it became apparent that predictive control methods display qualities that could be utilized in complex, nonlinear flight control applications [2]. Model predictive control, in general, has been identified as a method that offers good possibilities for reconfiguration and fault-tolerant control [1], [3]-[6]. This claim is simply due to model predictive control's ability of handling most of the challenges of reconfigurable control in a generic and systematic manner. The choice of NMPC over standard nonlinear control methods is therefore justified by the fact that they are not developed in order to handle constraints in a systematic way. In addition, many nonlinear methods depend on complicated design procedures that do not scale well to large systems [7].

Most fault tolerant control systems rely on or integrate fault detection and diagnosis (FDD) subsystems in order to

D. K. Kufoalor and T. A. Johansen are with the Department of Engineering Cybernetics, Norwegian University of Science and Technology, O.S. Bragstads plass 2D N-7491 Trondheim, Norway. kwame.kufoaloreitk.ntnu.no, tor.arne.johansenditk.ntnu.no accommodate component failures automatically. This work reveals further benefits of NMPC's limited reliance on external FDD systems and external reconfiguration mechanisms, compared to earlier indications in [3], which designed a model predictive controller for a linear stable aircraft model.

The work presented here deviates from the most common way of NMPC implementation. Predictive control in general is usually implemented on top of low-level controllers which take care of fast system dynamics and stabilization (if necessary) [2], [3]. In [8], NMPC is shown to provide better performance compared to a Linear Parameter Varying (LPV) control approach for the F-16 model used in the work presented here. The NMPC scheme in [8] was implemented on a prestabilized F-16 aircraft model, and it employs regulation of state perturbations to a set as its control objective. The present work, on the other hand, explores both fast inner-loop and relatively slow outer-loop NMPC autopilot functionalities for time varying references, and therefore demonstrates the powerful potentials of NMPC as capable of replacing the prestabilizing low-level controllers. This work focuses on the inherent fault tolerance of NMPC, and therefore reveals NMPC capabilities in the absence of a model reflecting severe actuator faults.

\section{THE AIRCRAFT MODEL}

In aircraft control design, it is a common practice to use decoupled equations of motion. The nonlinear equations of motion are based on formulations in [9].

The longitudinal equations are as follows:

$$
\begin{aligned}
\dot{h} & =V_{T} \cos \alpha \sin (\gamma+\alpha)-V_{T} \sin \alpha \cos (\gamma+\alpha) \\
\dot{V}_{T} & =\left(F_{T} \cos \alpha-D-\mathrm{m} g \sin \gamma\right) / \mathrm{m} \\
\dot{\gamma} & =\left(F_{T} \sin \alpha+L-\mathrm{m} g \cos \gamma\right) / \mathrm{m} V_{T} \\
\dot{\alpha} & =Q-\dot{\gamma} \\
\dot{Q} & =\mathscr{M} / I_{y}
\end{aligned}
$$

where $\mathrm{m}$ is the aircraft mass, $\mathscr{M}$ is the pitching moment, $I_{y}$ is the moment of inertia about the body $y$-axis, and $g$ is the gravitational acceleration. The drag force $D$ and the lift force $L$ are calculated from:

$$
\begin{aligned}
D & =-X_{T} \cos \alpha-Z_{T} \sin \alpha \\
L & =X_{T} \sin \alpha-Z_{T} \cos \alpha
\end{aligned}
$$

where $X_{T}$ and $Z_{T}$ are the total longitudinal and vertical aerodynamic forces respectively. The longitudinal differential states and parameters are described in tables I and II. 
TABLE I: NMPC flight path control design parameters and prediction model

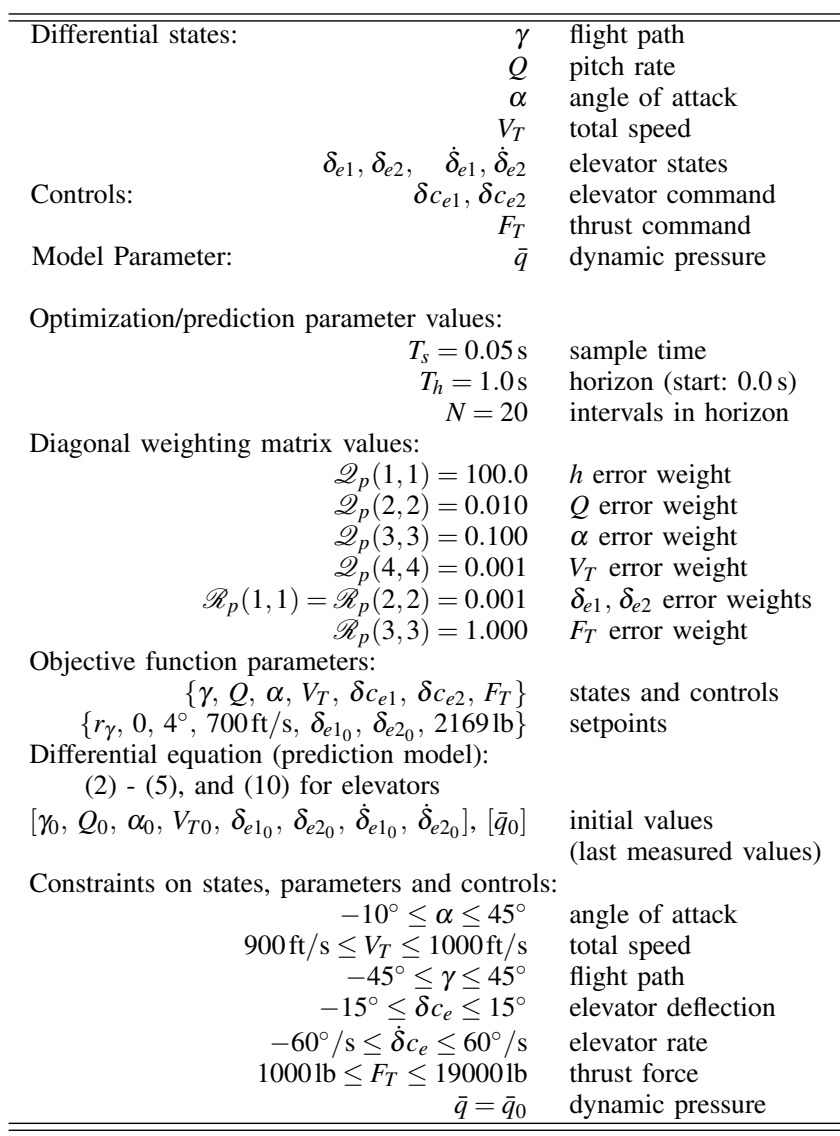

The equations of motion for the lateral channel assume constant longitudinal states $\alpha_{0}, V_{T 0}, \Theta_{0}$ (pitch angle):

$$
\begin{aligned}
\dot{\beta}= & P \sin \alpha_{0}-R \cos \alpha_{0} \\
& +\left(C-F_{T 0} \cos \alpha_{0} \sin \beta+\mathrm{m} g_{2}\right) / \mathrm{m} V_{T 0} \\
\dot{P}= & \mathscr{L} I_{z} /\left(I_{x} I_{z}-I_{x z}^{2}\right)+\mathscr{N} I_{x z} /\left(I_{x} I_{z}-I_{x z}^{2}\right) \\
\dot{R}= & \mathscr{L} I_{x z} /\left(I_{x} I_{z}-I_{x z}^{2}\right)+\mathscr{N} I_{x} /\left(I_{x} I_{z}-I_{x z}^{2}\right) \\
\dot{\Phi}= & P+R \tan \Theta_{0} \cos \Phi
\end{aligned}
$$

where $\mathscr{L}$ and $\mathscr{N}$ are the rolling and yawing moments respectively. $I_{x}$ and $I_{z}$ are, respectively, the moments of inertia about the body $x$ and $z$ axes, and $I_{x z}$ is the product of inertia. The gravity component $g_{2}$ takes the form:

$$
\begin{aligned}
g_{2}= & g\left(\cos \alpha_{0} \sin \beta \sin \Theta_{0}+\cos \beta \sin \Phi \cos \Theta_{0}\right. \\
& \left.-\sin \alpha_{0} \sin \beta \cos \Phi \cos \Theta_{0}\right)
\end{aligned}
$$

and the cross-wind force $C$ is calculated from:

$$
C=-X_{T 0} \cos \alpha_{0} \sin \beta+Y_{T} \cos \beta-Z_{T 0} \sin \alpha_{0} \sin \beta
$$

where $Y_{T}$ is the total transverse force. The lateral differential states and parameters are described in tables III and IV.

All the actuators of the control surfaces are modeled as first order dynamics with time constants $T=0.0495 \mathrm{~s}$ (which specify the bandwidths). That is,

$$
\dot{\delta}(t)=T^{-1}\left(\delta_{c}(t)-\delta(t)\right)
$$

TABLE II: NMPC altitude-hold (climb control) autopilot design parameters and prediction model

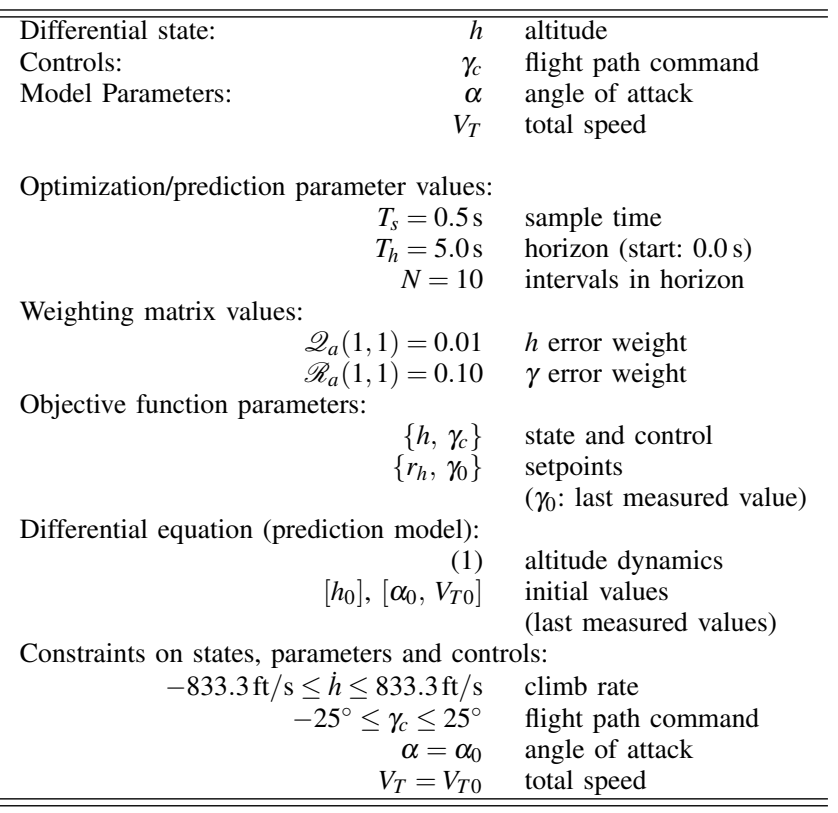

where $\delta_{c}$ is the commanded input and $\delta$ is the actual actuator deflection. The control surfaces are considered as the main control effectors in this work. The engine thrust force was therefore kept constant in all the test simulations.

The nonlinear mathematical model uses aerodynamic data from NASA-Langley wind tunnel tests on a subscale model of an F-16 aircraft [10]. The F-16 aerodynamic look-up tables and the coefficient equations used to sum up the contributions to the forces $X_{T}\left(\alpha, \beta, Q, \delta_{e}\right), Y_{T}\left(\alpha, \beta, P, R, \delta_{a}, \delta_{r}\right)$, $Z_{T}\left(\alpha, \beta, Q, \delta_{e}\right)$, and the moments $\mathscr{L}\left(\alpha, \beta, P, R, \delta_{a}, \delta_{e}, \delta_{r}\right)$, $\mathscr{M}\left(\alpha, \beta, Q, \delta_{e}\right), \mathscr{N}\left(\alpha, \beta, P, R, \delta_{r}, \delta_{a}, \delta_{e}\right)$, are also given in [9], [10]. The original (high fidelity) data cover $-20^{\circ}$ to $90^{\circ}$ angle of attack $(\alpha)$ range, and sideslip angle $(\beta)$ range from $-30^{\circ}$ to $30^{\circ}$. For simplicity and reduction of required computational power, the low fidelity data found in [9] were used in the NMPC autopilot prediction model calculations. The reduced aerodynamic data cover a $-10^{\circ} \leq \alpha \leq 45^{\circ}$ range, and the $\beta$ dependence is also approximated in some cases. However, the model constructed from the low fidelity data exhibits steady state flight trim conditions, and corresponding dynamic modes that are close to those of the full high fidelity data model [9].

\section{THE NMPC PROBLEM}

Model Predictive Control (MPC) is an advanced control methodology that uses a multivariable process model (internal model) to predict future system and control behavior. MPC using nonlinear process models is termed nonlinear MPC (NMPC). Typical reasons for using NMPC are that the process operates in several steady states with significantly different dynamics, or there are large disturbances that excite nonlinearities.

The formulation of the NMPC problem includes constraints on inputs (manipulated variables) and states (con- 
TABLE III: NMPC turn coordination (roll-angle hold) autopilot parameters and prediction model

\begin{tabular}{|c|c|}
\hline $\begin{array}{r}\beta \\
\Phi \\
P \\
R \\
\delta_{a 1}, \delta_{a 2}, \quad \dot{\delta}_{a 1}, \dot{\delta}_{a 2} \\
\delta_{r}, \dot{\delta}_{r}\end{array}$ & $\begin{array}{l}\text { sideslip } \\
\text { roll } \\
\text { roll rate } \\
\text { yaw rate } \\
\text { aileron states } \\
\text { rudder states }\end{array}$ \\
\hline $\begin{array}{r}\delta c_{a 1}, \delta c_{a 2} \\
\delta c_{r}\end{array}$ & $\begin{array}{l}\text { aileron command } \\
\text { rudder command }\end{array}$ \\
\hline $\begin{array}{r}\bar{q} \\
\alpha \\
V_{T} \\
\Theta \\
F_{T} \\
X_{T}, \quad Z_{T}\end{array}$ & $\begin{array}{l}\text { dynamic pressure } \\
\text { angle of attack } \\
\text { total speed } \\
\text { pitch } \\
\text { thrust force } \\
\text { total force in } X, Z\end{array}$ \\
\hline $\begin{array}{l}\text { Optimization/prediction parameter values: } \\
\qquad \begin{array}{r}T_{s}=0.05 \mathrm{~s} \\
T_{h}=1.0 \mathrm{~s} \\
N=20\end{array}\end{array}$ & $\begin{array}{l}\text { sample time } \\
\text { horizon (start: } 0.0 \mathrm{~s} \text { ) } \\
\text { intervals in horizon }\end{array}$ \\
\hline $\begin{aligned} \text { Diagonal weighting matrix values: } & \\
\mathscr{Q}_{t}(1,1) & =1.00 \\
\mathscr{Q}_{t}(2,2) & =10.0 \\
\mathscr{Q}_{t}(3,3) & =1.00 \\
\mathscr{Q}_{t}(4,4) & =1.00 \\
\mathscr{R}_{t}(1,1)=\mathscr{R}_{t}(2,2) & =0.05 \\
\mathscr{R}_{t}(3,3) & =0.10\end{aligned}$ & $\begin{array}{l}\beta \text { error weight } \\
\Phi \text { error weight } \\
P \text { error weight } \\
R \text { error weight } \\
\delta_{a 1}, \delta_{a 2} \text { error weights } \\
\delta c_{r} \text { error weight }\end{array}$ \\
\hline $\begin{array}{l}\text { Objective function parameters: } \\
\qquad \begin{array}{r}\left\{\beta, \Phi, P, R, \delta c_{a 1}, \delta c_{a 2}, \delta c_{r}\right\} \\
\left\{0, r_{\Phi}, 0,0, \delta_{a 1_{0}}, \delta_{a 2_{0}}, \delta_{r 0}\right\}\end{array}\end{array}$ & $\begin{array}{l}\text { states and controls } \\
\text { setpoints }\end{array}$ \\
\hline $\begin{array}{l}\text { Differential equation (prediction model): } \\
(6) \text { - (9), and (10) for ailerons and rudder } \\
{\left[\begin{array}{r}{\left[\beta_{0}, \Phi_{0}, P_{0}, R_{0}, \delta_{a 1_{0}}, \delta_{a 2_{0}}, \dot{\delta}_{a 1_{0}}, \dot{\delta}_{a 2_{0}}, \delta_{r 0}, \dot{\delta}_{r 0}\right]} \\
{\left[\bar{q}_{0}, \alpha_{0}, V_{T 0}, \Theta_{0}, F_{T 0}, X_{T 0}, Z_{T 0}\right]}\end{array}\right.}\end{array}$ & $\begin{array}{l}\text { initial values: } \\
\text { for states } \\
\text { for parameters } \\
\text { (last measured values) }\end{array}$ \\
\hline $\begin{array}{l}\text { Constraints on states, parameters and controls: } \\
\qquad \begin{array}{r}-308^{\circ} / \mathrm{s} \leq P \leq 308^{\circ} / \mathrm{s} \\
-15^{\circ} \leq \delta c_{a} \leq 15^{\circ} \\
-80^{\circ} / \mathrm{s} \leq \dot{\delta} c_{a} \leq 80^{\circ} / \mathrm{s} \\
-15^{\circ} \leq \delta c_{r} \leq 15^{\circ} \\
-120^{\circ} / \mathrm{s} \leq \dot{\delta} c_{r} \leq 120^{\circ} / \mathrm{s} \\
\alpha=\alpha_{0}, \quad V_{T}=V_{T 0} \\
\alpha=\bar{q}_{0}, \quad F_{T}=F_{T 0} \\
\Theta X_{T}=X_{T 0}, \quad Z_{T}=Z_{T 0}\end{array}\end{array}$ & $\begin{array}{l}\text { roll rate } \\
\text { aileron deflection } \\
\text { aileron rate } \\
\text { rudder deflection } \\
\text { rudder rate } \\
\text { dynamic pressure } \\
\text { angle of attack, speed } \\
\text { pitch, thrust force } \\
\text { total force in } X, Z\end{array}$ \\
\hline
\end{tabular}

trolled variables). The formulated problem is then solved by using mathematical programming to optimize predicted future performance at each sampling interval.

Since NMPC relies on its internal model for predictions, it will require a fault model of the system if the severity of the damage is such that the dynamics of the system changes.

\section{A. Formulation of the NMPC problem}

A very general and simple formulation of the nonlinear model predictive problem has the control objective of minimizing a cost function $J$, which takes the form:

$$
J(x[0, T], u[0, T])=\int_{0}^{T} \ell(x(t), u(t), t) \mathrm{d} t
$$

where $\ell(x(t), u(t), t)$ is a non-negative function, termed the stage cost, and $T>0$ is the horizon. The stage cost $\ell($.$) is$ chosen as an $l_{2}$ type cost function [7]:

$$
\ell(x(t), u(t), t)=\left\|x(t)-r_{x}(t)\right\|_{\mathscr{Q}}^{2}+\left\|u(t)-r_{u}(t)\right\|_{\mathscr{R}}^{2}
$$

TABLE IV: NMPC heading-hold autopilot parameters and prediction model

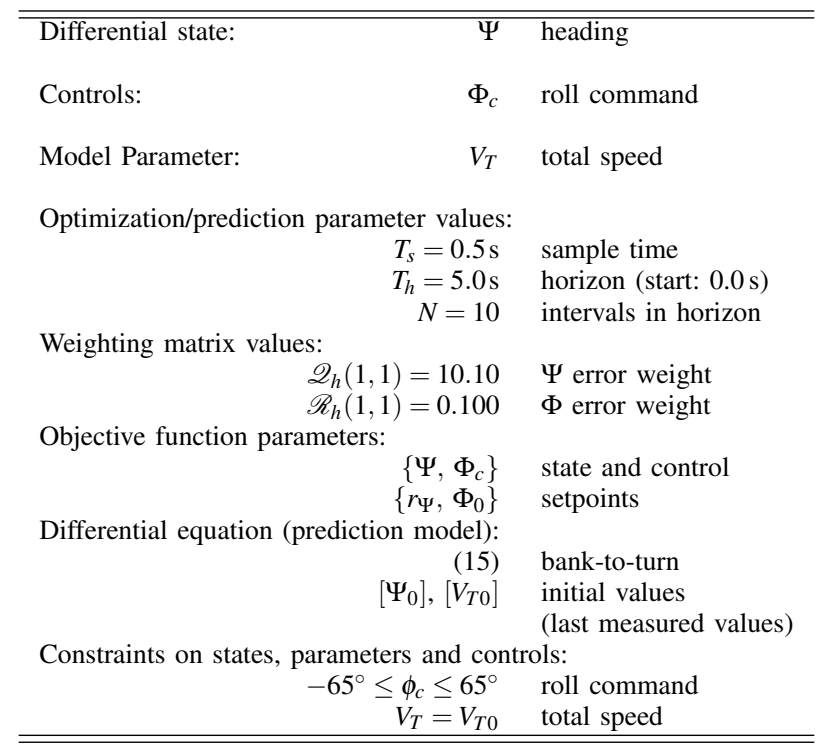

where the properties of the weight matrices $\mathscr{Q} \geq 0$ and $\mathscr{R} \geq 0$ are essential for performance, and in some cases also stabilty. The plant to be controlled has a state vector $x$ (with setpoint $r_{x}$ ), an input vector $u$ (with setpoint $r_{u}$ ), and a nonlinear behavior governed by the vector differential equation

$$
\dot{x}=f(x, u)
$$

In addition to the equality constraints introduced by the nonlinear plant model (12), general inequality constraints jointly on states and inputs can be defined as

$$
g(x(t), u(t), t) \leq 0
$$

and finally, the control input vector is constrained to some set $u(t) \in U$, where

$$
U=\left\{u_{\min } \leq u(t) \leq u_{\max }\right\}
$$

and $u_{\min }, u_{\max }$ are, respectively, the minimum and maximum limits of $u(t)$.

In general, stability is not guaranteed for the simple NMPC formulation above, since the horizon is finite. Several approaches have therefore been proposed, introducing modifications such as terminal sets and constraints to ensure stability [11], [12]. However, stability enforcing terminal constraints may be relaxed, or even skipped completely, since they tend to be conservative and often not needed when the NMPC is carefully designed [7]. In this work, the NMPC autopilot design approach, the choice of horizons, and other design parameters resulted in the generation of reliable control trajectories, without the need of modifying the NMPC formulation. See [13] for further details, and [8] for a stability guaranteeing modification for the F-16 model.

Another key aspect of NMPC is the understanding of how design parameters such as the horizon, the weight matrices, and the time constant of the reference trajectory are tuned. The reference trajectory defines an ideal trajectory from the 
plant's current output to the desired set point, and therefore defines an important aspect of the closed-loop behavior of the NMPC scheme used in this work.

\section{NMPC AUTOPILOTS}

\section{A. Longitudinal Motion Control}

An altitude-hold autopilot which relies on a flight path controller was implemented for maneuvers in the longitudinal channel. In a classical autopilot design sense, the altitude control is seen as the (slower) outer loop while the flight path control is the (faster) inner loop. The measured states of the flight path NMPC problem were introduced as time-varying parameters in the altitude NMPC problem formulation. Design details are shown in tables I and II. The design structure allows individual sampling times and horizons to be employed, reflecting the relative responses and sampling rates required for each autopilot dynamics.

\section{B. Lateral Motion Control}

The NMPC lateral autopilots include a Heading-hold controller, which performs coordinated turns using a roll-angle hold controller. The measured states of the turn-coordination (roll-angle hold) autopilot were introduced as time-varying parameters in the heading-hold NMPC problem formulation.

The following nonlinear bank-to-turn equation was used to specify the desired behavior of the aircraft during a turn maneuver.

$$
\dot{\Psi}=g \sin \left(\Phi_{c}\right) / V_{T}
$$

It states that a roll angle $\Phi_{c}$ different from zero will induce a heading rate $\dot{\Psi}$, which turns the aircraft. Design details are shown in tables III and IV, and the complete design process can be found in [13].

\section{SOLVING THE NMPC PROBLEM}

Many existing approaches to solving the NMPC problem are based on Sequential Quadratic Programming (SQP) methods, which make quadratic approximations to the objective function and linear approximations to the constraints, and iteratively solve a Quadratic Program (QP) to find the search direction leading to the optimal solution [7], [14].

The optimization package used in solving the NMPC problems formulated in this work is the ACADO Toolkit. Section V-A highlights the key features of ACADO and the optimizer parameters used in this work.

\section{A. NMPC optimization solver: ACADO for MATLAB}

ACADO Toolkit is an algorithm collection for automatic control and dynamic optimization, implemented as selfcontained $\mathrm{C}++$ code. The NMPC autopilots were implemented in two parts. The first consisting of the optimization problem, composed in MATLAB, and the second being the NMPC prediction model implemented as C-code.

The autopilot optimization problems were solved using ACADO's multiple-shooting SQP-type method combined with Runge-Kutta integrator (of order 4/5) for the state integration. Multiple-shooting is a discretization-type parameter option in ACADO. The KKT tolerance (Karush-KuhnTucker first order (necessary) optimality conditions), which is used for the convergence criterion of the SQP algorithm, was set to $10^{-6}$ (default value in ACADO). The default value of the maximum number of iterations (1000) was also maintained for all simulations and tests. The maximum number of iterations offers a means of specifying a trade off between result accuracy and computational time. Smaller values will reduce the execution time, but the result could be far from optimal. Details about the ACADO Toolkit can be found in [15], [16].

\section{TEST CASES AND RESULTS}

This section focuses on using control surface (or actuator) fault simulations to primarily verify the fault tolerant capabilities of NMPC as a reconfigurable controller.

The simulations were initialized at trim conditions where $h_{0}=20000 \mathrm{ft}, V_{T 0}=500 \mathrm{ft} / \mathrm{s}, \alpha_{0}=5.53^{\circ}, F_{T 0}=2168.71 \mathrm{lb}$, $\delta_{e 1_{0}}=\delta_{e 2_{0}}=-2.77^{\circ}$, and $\delta_{a 1_{0}}=\delta_{a 2_{0}}=\delta_{r 0}=0^{\circ}$. The test maneuvers began with a $20^{\circ}$ heading change demand to the heading and turn coordination autopilots, while the altitude autopilot was commanded to maintain $20000 \mathrm{ft}$. The altitude set point was changed to $20200 \mathrm{ft}$ after $20 \mathrm{~s}$, and the heading demand and was stepped down to $10^{\circ}$ after $30 \mathrm{~s}$. The main objective is to stabilize the aircraft and perform turn and climb maneuvers as well as attaining straight level flight in the presence of control surface faults.

The results for three test cases are presented. Case 1 assumes the existence of an FDD system that updates the NMPC autopilot with new control surface position limits or rate limits when faults occur (according to the FDD scheme in [17]), but it does not implement any external reconfiguration mechanism. Case 2 assumes no FDD system exists, and therefore simulates the case where the control system relies on only the inherent fault detection and reconfigurable capabilities of NMPC (enabled through the effective use of measured actuator position feedback). Case 3 assumes the existence of an FDD system and, in addition, employs the following simple reconfiguration mechanism.

1) for each actuator, scan for locked/jammed actuator by checking for changes in lower and upper limits (worst case $\delta_{\text {min }}=\delta_{\text {max }}$ )

2) if an actuator is locked, decrease the weight of all the 'healthy' actuators in the same channel (longitudinal or lateral), by a predefined factor.

3) finally, reduce the weight on any secondary objectives that share the same remaining 'healthy' actuators (that are capable of achieving similar effects as the jammed actuator), by a predefined factor.

The tests were performed while the left aileron was locked at $-15^{\circ}$. The results of the three test cases are compared in figure 1. Apart from differences noticed in the rudder input actions (figure 1e) and their accompanying sideslip trajectories (in figure 1c), the trajectories of Case 1 and Case 2 are close. In Case 2, the NMPC turn coordination controller quickly realizes through measured actuator position feedback 
(implemented as initial values) that the left aileron is not responding to its commands. In other words, the controller perceives a situation similar to when it has driven the aileron to its limit, and starts to move other control surfaces. Not using any information about the failure situation from an FDD system, the results of Case 2 can be considered as good, compared to those of Case 1 and Case 3.

It can be emphasized that in Case 1 , the locked aileron fault was reported to the controller (by setting $\delta_{a 1_{\min }}=$ $\delta_{a 1_{\text {max }}}=-15^{\circ}$ ) and, in addition, the weights (error penalties) were adjusted in Case 3. The weights on keeping set-points for sideslip $\left(\mathscr{Q}_{t}(1,1)\right)$, roll rate $\left(\mathscr{Q}_{t}(3,3)\right)$, and yaw rate $\left(\mathscr{Q}_{t}(4,4)\right)$ were reduced by a factor of 10 , to further prioritize the use of roll angle to achieve the desired heading. The same reduction was made on the weight for the right aileron $\left(\mathscr{R}_{t}(2,2)\right)$, with the intention of making it more active.

Another interesting result was obtained when the weight on set-point error for heading was reduced from 10 to 2.5. The intention was to relax the objective of fast control from a guidance system point of view. The results, compared to the fault-free aileron test, are shown in figure 2. The performance improvement indicates that some reduction in the overall performance objective that reflects the fault situation may be useful in the event of an extreme control surface failure in order to achieve graceful degradation of performance.

Test cases that examine free-play (float-type) failures and significant changes in actuator position or rate limits are documented in [13]. Test results show that these failures have very little effect on NMPC performance, since actuator constraints are handled effectively as part of NMPC's primary objectives. The free-floating type failure is also rather mild compared to the locked actuator or hard-over failure tests, since no significant disturbance is introduced by the 'floating' control surface.

Simulations of the complete test setup, including all four autopilots and the fully coupled nonlinear F-16 model (plant), were done on a single core of a computer with Intel Core i7-3720QM 2.6GHz processor and 8GB RAM. Average computational times of $0.004 \mathrm{~s}, 0.006 \mathrm{~s}, 0.093 \mathrm{~s}$, and $0.044 \mathrm{~s}$ were recorded for the heading-hold, altitudehold, turn coordination, and flight path control autopilots respectively.

\section{CONCLUSIONS}

This work illustrates the elegance of combining multivariable, nonlinear, and constrained controller behavior into a well structured and powerful NMPC framework in order to implement reconfigurable fault tolerant control.

Fault tolerance of NMPC has been shown, through autopilot implementation results, to be achievable through an effective combination of fault detection capabilities and reconfiguration. Observations made indicate a clear tendency of NMPC to effectively attempt to accomplish the set objectives, even with no explicit identification of the fault.

It can be further argued that in cases where fault detection is not available or the available FDD system is not reliable, fault tolerant graceful degradation of performance is achievable when the NMPC utilizes its inherent robustness.

Real-time implementation is known as a challenging aspect of NMPC. However, using fast NMPC solvers and/or hardware with adequate computational capacity, real-time implementation of the NMPC autopilots is possible. Moreover, the proposed autopilot formulations allow implementation in separate tasks on a multi-core computer or on separate processors.

\section{ACKNOWLEDGMENT}

We thank Åge Skullestad and his colleagues at Kongsberg Defence Systems for their inputs on autopilot specifications, and for useful discussions on fighter aircraft dynamics.

\section{REFERENCES}

[1] Y. Zhang and J. Jiang, "Bibliographical review on reconfigurable faulttolerant control systems," Annual Reviews in Control, no. 32, pp. 229252, 2008.

[2] T. Keviczky and G. J. Balas, "Receding Horizon Control of F-16 Aircraft: A Comparative Study," Control Engineering Practice, vol. 14, no. 9, pp. 1023-1033, 2006.

[3] J. M. Maciejowski, Predictive Control: with constraints. Pearson and Prentice Hall, 2002.

[4] J. M. Maciejowski and C. N. Jones, "MPC fault-tolerant flight control case study: Flight 1862," in Proceedings of the 5th IFAC symposium on fault detection, supervision and safety for technical processes. IFAC, June 2003, pp. 121-126.

[5] C. N. Jones and P. College, "Reconfigurable Flight Control, First Year Report," Department of Engineering, University of Cambridge, Tech. Rep., March 2005.

[6] M. M. Kale and A. J. Chipperfield, "Reconfigurable Flight Control Strategies Using Model Predictive Control," in Proceedings of the 2002 IEEE International Symposium on Inteligent Control. Vancouver, Canada: IEEE, October 2002, pp. 43-48.

[7] T. A. Johansen, "Introduction to Nonlinear Model Predictive Control and Moving Horizon Estimation," in Selected Topics on Constrained and Nonlinear Control. STU/NTNU, 2011, ch. 1, pp. 1-29, iSBN 978-80-968627-4-0.

[8] R. Bhattacharya, G. J. Balas, M. A. Kaya, and A. Packard, "Nonlinear Receding Horizon Control of F-16 Aircraft," Journal of Guidance, Control, and Dynamics, vol. 25, no. 5, pp. 924-931, 2002.

[9] B. L. Stevens and F. L. Lewis, Aircraft Control and Simulation. Wiley, 2003, ch. 1 to 5, pp. 1-508.

[10] L. T. Nguyen, M. E. Ogburn, W. P. Gilbert, K. S. Kibler, P. W. Brown, and P. L. Deal, "Simulator Study of Stall/Post-Stall Characteristics of a Fighter Airplane with Relaxed Longitudinal Static Stability," NASA Scientific and Technical Information Branch, Langley Research Center, Hampton, Virginia, Tech. Rep. 1538, December 1979.

[11] H. Chen and F. Allgöwer, "A Quasi-Infinite Horizon Nonlinear Model Predictive Control Scheme with Guaranteed Stability," Automatica, vol. 34, no. 10, pp. 1205-1217, 1998.

[12] D. Q. Mayne, J. B. Rawlings, C. V. Rao, and P. O. M. Scokaert, "Constrained model predictive control: Stability and optimality," $\mathrm{Au}$ tomatica, vol. 36, pp. 789-814, 2000.

[13] D. K. Kufoalor, "Reconfigurable Autopilot Design using Nonlinear Model Predictive Control: Application to High Performance and Autonomous Aircraft," Master's thesis, Department of Engineering Cybernetics, Norwegian University of Science and Technology (NTNU), June 2012.

[14] J. Norcedal and S. J. Wright, Numerical Optimization. Springer, 2006 , ch. 18 , pp. 529-560.

[15] B. Houska, H. J. Ferreau, and M. Diehl, "ACADO Toolkit - An Open Source Framework for Automatic Control and Dynamic Optimization," Optimal Control Applications and Methods, vol. 32, no. 3, pp. 298312, 2011.

[16] D. Ariens, B. Houska, and H. J. Ferreau, "ACADO for Matlab User's Manual," http://www.acadotoolkit.org, 2010-2011.

[17] N. Swain and S. Manickavasagar, Fault Tolerant Flight Control, A Benchmark Challenge. SpringerLink, 2010, ch. 14, pp. 399-422. 

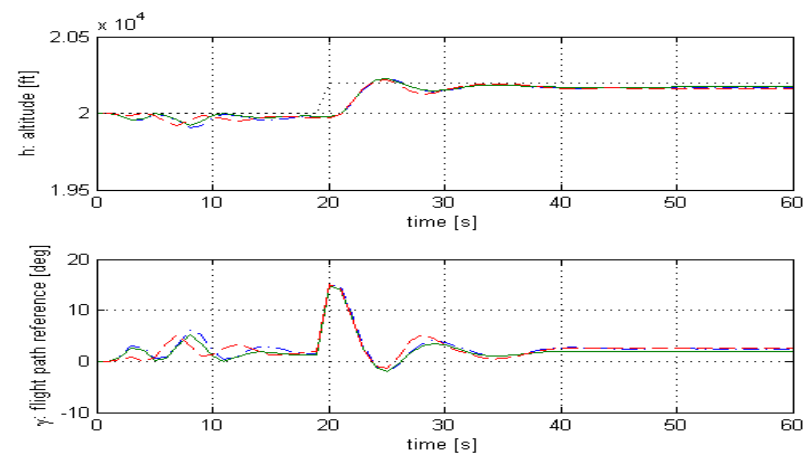

(a) Altitude and flight path control
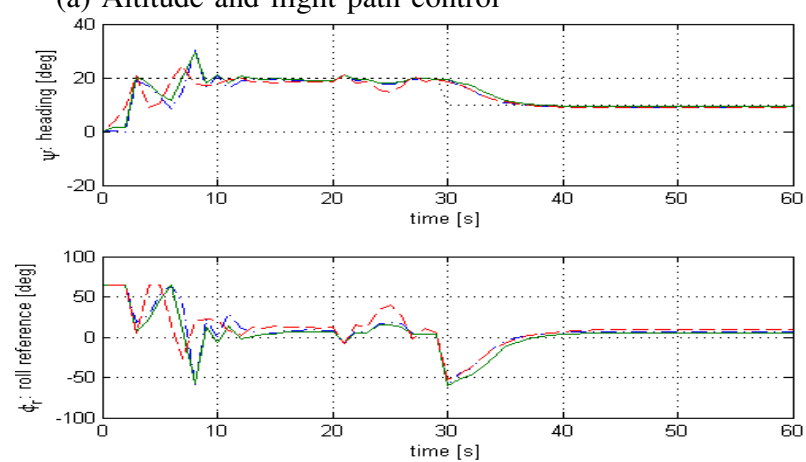

(b) Heading and bank-to-turn control
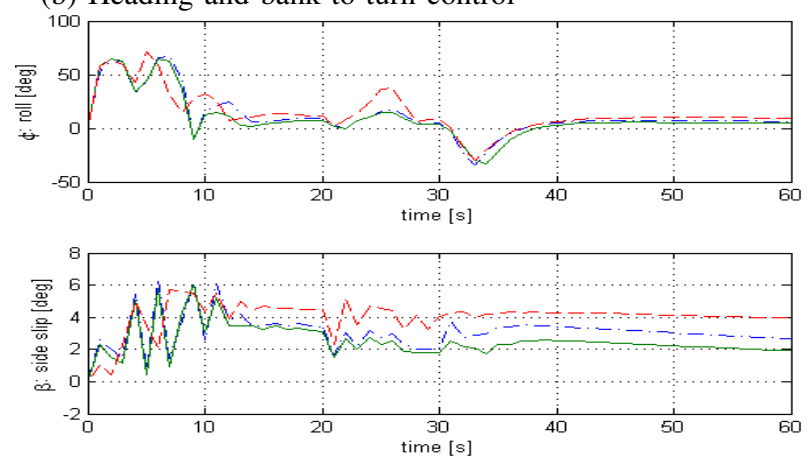

(c) Roll and sideslip

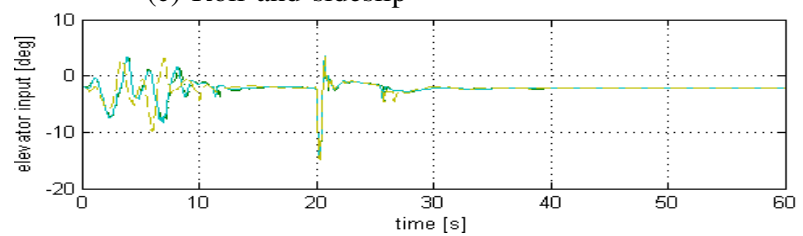

(d) Elevator input
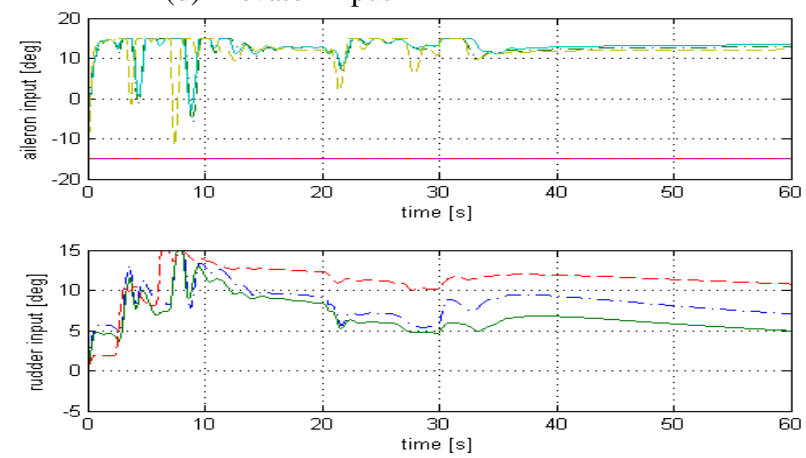

(e) Aileron and rudder inputs

Fig. 1: NMPC fault tolerance: F-16 left aileron $\left(\delta_{a 1}\right)$ locked at $-15^{\circ}$. Case $1(-\cdot)$, Case $2(-)$, Case $3(--)$.
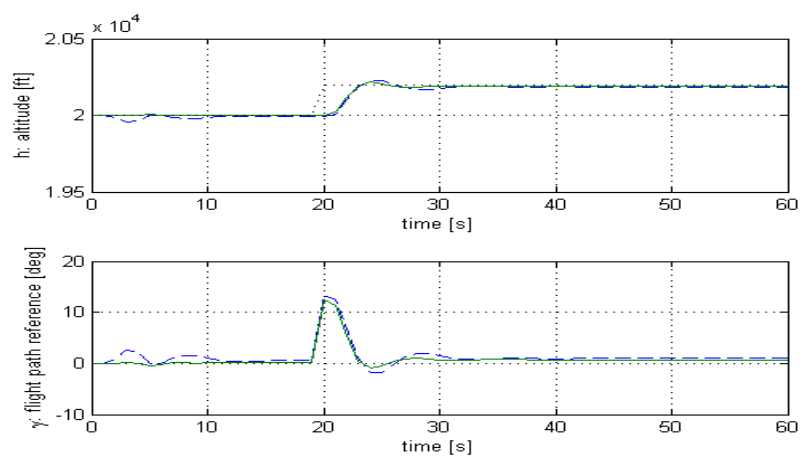

(a) Altitude and flight path control
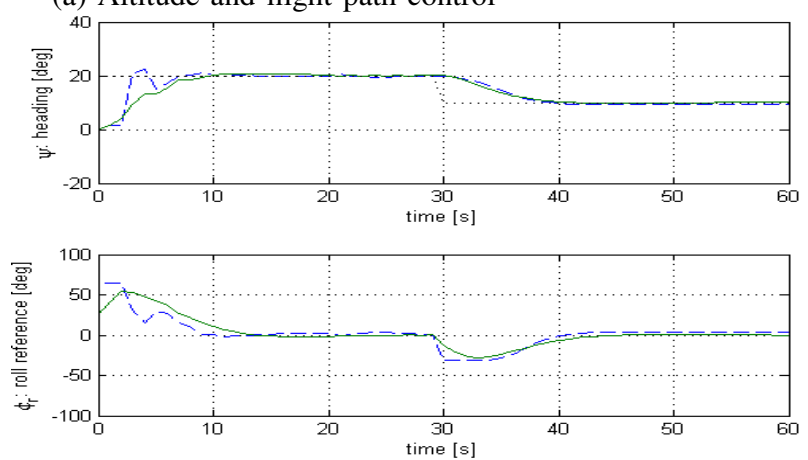

(b) Heading and bank-to-turn control
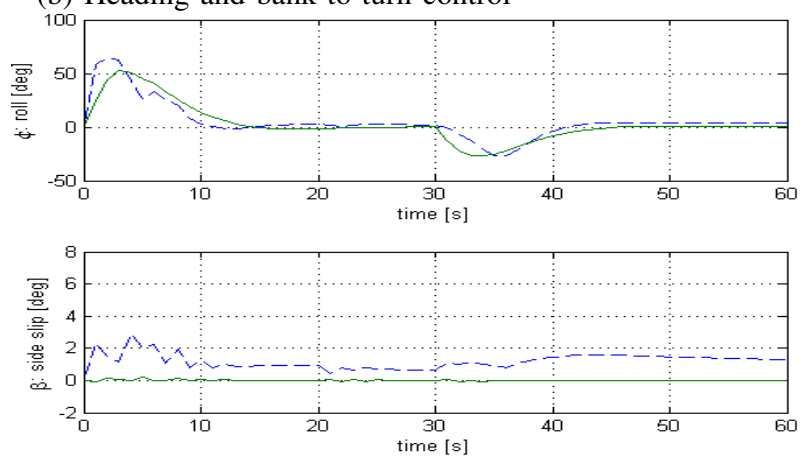

(c) Roll and sideslip

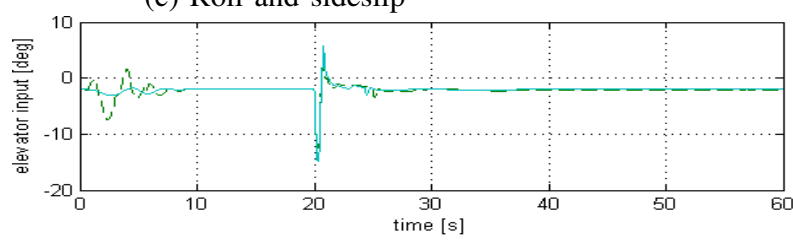

(d) Elevator input
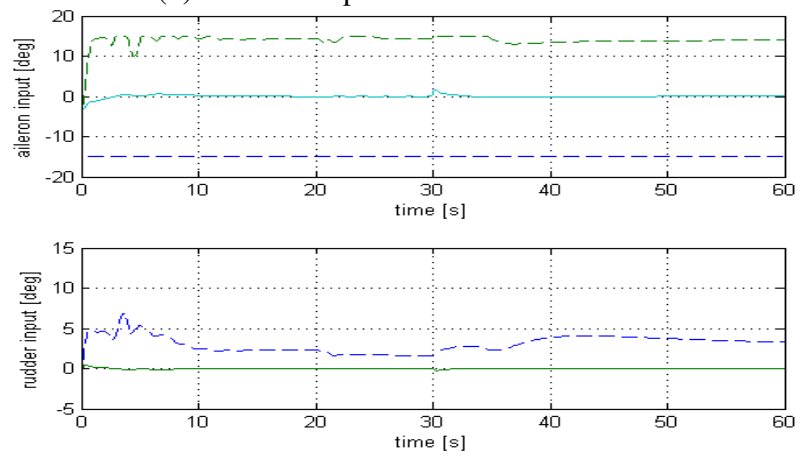

(e) Aileron and rudder inputs

Fig. 2: Relaxed heading control objective. Left aileron $\left(\delta_{a 1}\right)$ locked at $-15^{\circ}$ (Case $2--$ ). Fault-free aileron test (一). 